\title{
Improved dyeability of polypropylene fabrics finished with $\beta$-cyclodextrin-citric acid polymer
}

\author{
Yassine El Ghoul ${ }^{1}$, Bernard Martel ${ }^{1}$, Ahmida El Achari ${ }^{2}$, Christine Campagne ${ }^{2}$, Lova Razafimahefa ${ }^{2}$ \\ and Isabelle Vroman ${ }^{2}$
}

Polypropylene (PP)-knitted fabrics were finished with $\beta$-cyclodextrin ( $\beta$-CD) and crosslinked with citric acid (CTR). The polyCTR$\beta$-CD polymer-coated fibers were wash resistant. We report that the rate of immobilized $C D$ was controlled by temperature and curing time. The weight increase of the fabrics after modification with native $\beta$-CD and CTR reached $30 \% w t$. The treated PP supports were dyed using disperse, acid and reactive dyes. Standard tests of wash fastness (shade change and staining) were conducted and showed that all dyes were fixed, because of inclusion complexation with immobilized cavities on the one hand and sorbed hydrogen and ionic bonds that occurred between the polyCTR- $\beta$-CD coating and the tested dyestuffs on the other. Polymer Journal (2010) 42, 804-811; doi:10.1038/pj.2010.80

Keywords: citric acid; cyclodextrins; dyeing; polypropylene; textile finishing; wash fastness

\section{INTRODUCTION}

Cyclodextrins (CDs) are cyclic oligosaccharides commonly composed of six to eight glucopyrannose units $(\alpha, \beta$ and $\gamma$-CDs) that have the overall shape of a truncated cone. Because of their relatively hydrophobic interior cavity, CDs have the ability to form inclusion complexes with a wide range of volatile substrates in the gaseous phase, or with partially hydrophobic compounds dissolved and solubilized in water. This property of CDs has led to their application in various areas such as enzyme mimics, catalysis and the encapsulation of active substances, flavoring agents, fragrances, pesticides and drugs. For these reasons, the application of CDs and their derivatives in the textile field appeared in the early 1980 s. $^{1,2}$

The dyeability of fibers is controlled by both their chemical and physical properties. Fibers that have polar functional groups in their molecular repeat units can be dyed more easily. These polar groups may serve as active sites for chemical bonding with dye molecules. Because PP molecular chains have no polar functional groups, the dye molecules cannot be chemically attracted to fibers. Moreover, dye molecules cannot be strongly adsorbed by fiber surfaces because of their hydrophobic properties. Therefore, these adsorbed molecules, which interact with the fiber surface by weak Van der Waal's forces, are easily washed off.

Fiber modifications have been investigated to make polypropylene (PP) dyeable. These include copolymerization to provide dye-compatible sites along the macromolecular chain and blending PP with dyeable polymers. ${ }^{3,4}$ These methods have, however, generally led to a significant decrease in the desirable physical properties of PP fibers.
In recent years, surface modification of hydrophobic fibers by plasma treatment has opened up new possibilities in this field. Functional groups can be introduced onto the surface of PP fibers by special gas plasma treatment or plasma polymerization. ${ }^{5}$

Previous reports deal with the use of CDs as dyeing auxiliaries that improve dye sorption onto fibers, because of the increased solubility and affinity of the CD-dye inclusion complex with fibers. ${ }^{6,7}$

Other reports investigate a different approach that consists of preliminary modification of textiles with $\mathrm{CDs}$ before the dyeing process. This later approach was exclusively possible in cellulosic or proteinic fibers, as most textiles that finish with $\mathrm{CDs}$ were only applicable in fibers carrying sufficient hydroxyl or amino groups available for reaction with the crosslinking agent or with the reactive group of the CD's derivates. ${ }^{8,9}$

Besides, in 2000, we proposed a method of grafting CDs onto PP through a process involving the use of electron beam irradiation. ${ }^{10}$ Thereafter, in 2002, we proposed a chemical path based on the use of polycarboxylic acids, which was not only efficient for cotton and wool modification ${ }^{11}$ but also for polyester, ${ }^{12}$ polyamide ${ }^{13}$ and PP fibers. ${ }^{14}$ It was observed that the crosslinking reaction between polycarboxylic acids and CDs occurred in situ in the fiber network and resulted in the coating of fibers by a crosslinked CD polymer.

In this paper, we first report the results of a knitted PP fabric finishing with $\beta-\mathrm{CD}$, and the optimization of various parameters such as temperature and curing time. In a second part, we present the use of various types of dyes in dyeing experiments of these $\beta$-CD-modified PP fabrics.

\footnotetext{
${ }^{1}$ UMET, Engineering of Polymeric Systems Group, University of Lille 1, Sciences and Technologies, Villeneuve d'Ascq cedex, France and ${ }^{2}$ Laboratory of Textile Process and Materials, UPRES EA 2161, Research Unit of ENSAIT, Roubaix cedex 01, France

Correspondence: Professor B Martel, UMET, Engineering of Polymeric Systems Group, University of Lille 1, Sciences and Technologies, Cité scientifique, Batiment C6, Villeneuve d'Ascq cedex 59655, France.

E-mail: bernard.martel@univ-lille1.fr

Received 18 December 2009; revised 19 July 2010; accepted 21 July 2010
} 


\section{EXPERIMENTAL PROCEDURE}

\section{Products}

PP yarn was supplied by SO.TU.FIL.D (Monastir, Tunisia) and knitted at ENSAIT with Dubied (Couvet, Switzerland) knitting machinery. $\beta-\mathrm{CD}$ was purchased from Roquette (Kleptose, Lestrem, France). Citric acid (CTR) and sodium dihydrogen hypophosphite were from Aldrich chemicals (Saint Quentin Fallavier, France). Disperse dye (Blue Artisil BSQ) and acid dye (Red Nylosane E-BM) were purchased from Clariant (Mutenz, Switzerland). Reactive dye (Yellow LANASOL 4G) was purchased from Ciba (Bâle, Switzerland).

\section{B-CD finishing}

PP-knitted fabrics were washed with soxhlet extractors and dried to eliminate industrial finishing products, especially sizing agents. Thereafter, the fabrics were treated with a pad-dry process using a horizontal two-roll padder and a thermofixation oven (Roaches, Leek, UK). The padding liquor was an aqueous solution containing $\mathrm{CTR} / \mathrm{NaH}_{2} \mathrm{PO}_{4} / \beta-\mathrm{CD}$ in a $10 / 3 / 10$ ratio, corresponding to the amount in grams of each reactant for $100 \mathrm{ml}$ of solution. Impregnated fabrics were then roll-squeezed, dried and thermofixed (at various temperatures and times) and finally washed with hot water by a soxhlet extractor. Raw and treated samples were dried for $30 \mathrm{~min}$ at $104^{\circ} \mathrm{C}$ before being weighed. The weight gain of the fabrics (symbolized by \%wt) was measured to evaluate the yield of the functionalization reaction and calculated by the following equation: $\% \mathrm{wt}=\left(m_{\mathrm{f}}-m_{\mathrm{i}}\right) / m_{\mathrm{i}} \times 100$, where $m_{\mathrm{i}}$ and $m_{\mathrm{f}}$ are the weight of the sample before and after treatment, respectively. The precision of the weight gain measurements was $\pm 1.5 \%$ wt.

\section{Characterization}

The surface characteristics of the fibers were determined with a Cahn balance with glycerol (surface energy of $63.4 \mathrm{~mJ} \mathrm{~m}^{-2}$ ). The contact angle $(\theta)$ measurement between the substrate and various liquids with known surface energy permits one to evaluate their surface tension. ${ }^{15}$

The following formula is used:

$$
\cos \theta=\frac{m g}{2 \pi r_{\mathrm{f}} \gamma}
$$

where $\theta$ indicates contact angle, $\gamma$ indicates surface energy of the solvent, $g$ is gravity, $m$ is the weight of the fiber and $r_{\mathrm{f}}$ is the ray of the fiber section.

In the present case, $r_{\mathrm{f} \text { moy }}=38.5 \mu \mathrm{m}$.

\section{Complexation study}

As it was not possible to evidence the inclusion complex between dyes and $\mathrm{CD}$ cavities fixed onto the fibers (for instance, by solid nuclear magnetic resonance spectroscopy), we carried out this investigation in solution. This approach was considered pertinent as it displayed the possible inclusion of dyes into $\beta$-CD cavities.

Therefore, interactions between $\beta$-CD and dyes were observed by ultravioletvisible spectrophotometry by mixing solutions of $\beta-C D$ and dyes. As we previously reported, ${ }^{16}$ a spectral change of a dye solution on addition of $\mathrm{CD}$ can be interpreted as evidence of host-guest complexation.

A concentrated dye solution was prepared beforehand (dispersed, acid and reactive dye: $2.10^{-5} \mathrm{M}$; solution $\mathrm{A}$ ). In parallel, another solution was prepared containing a $1 / 1000$ molar ratio $($ dye $) /(\beta-C D)$ (dye concentration $=2.10^{-5} \mathrm{M}$; solution B). Both solutions were agitated for 30 min under magnetic stirring and then submitted to ultrasonication for $15 \mathrm{~min}$. A volume of $50 \mathrm{ml}$ of various solutions was then prepared by adjusting molar ratios $\beta-\mathrm{CD} /$ dye from 0 to 900 by diluting solution $\mathrm{B}$ in adequate proportions with solution A. After $30 \mathrm{~min}$ of stirring, the visible absorption spectrum of the various complexes was measured. The Benesi-Hildebrand method ${ }^{17}$ allowed us to calculate the different constants of complexation between each dye and $\beta-\mathrm{CD}$ in solution. This method determines, for a fixed wavelength, the difference in absorbance between each dye/CD solution spectrum and the spectrum of solution $\mathrm{A}$ (this difference is expressed as $\Delta$ Abs). By plotting $1 / \mathrm{dA}$ versus $1 /(\mathrm{CD})$, it was possible to determine the constant of complexation from the slope and the $y$ intercept of this linear plot.

$$
\frac{[\mathrm{CD}]_{0} \cdot[\mathrm{S}]_{0}}{\Delta \mathrm{Abs}}=\frac{1}{K_{\mathrm{f}} \cdot \Delta \varepsilon}+\frac{[\mathrm{CD}]_{0}+[\mathrm{S}]_{0}}{\Delta \varepsilon}
$$

Table 1 Structure of the different dyes used in our study

\begin{tabular}{lll}
\hline Dyes & Class & Structure \\
\hline Blue Artisil & Disperse & dye \\
$\mathrm{BSQ}$ & &
\end{tabular}

$\begin{array}{ll}\text { Red Nylosane } & \text { Acid } \\ \text { E-BM } & \text { dye }\end{array}$

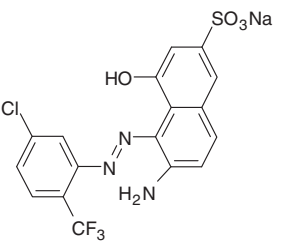

Yellow Reactive

LANASOL $4 G$ dye

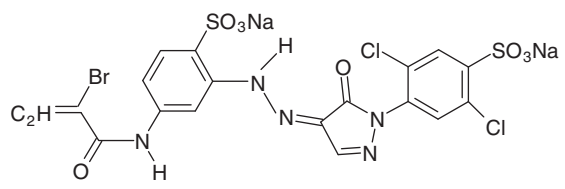

where $[\mathrm{CD}]_{0}$ indicates the concentration of $\mathrm{CD},[\mathrm{S}]_{0}$ indicates the concentration of dye, $\Delta \mathrm{Abs}$ is the difference of absorbance, $\Delta \varepsilon$ is the difference of wavelengths and $\mathrm{K}_{f}$ is the constant of complexation.

\section{Dyeing experiments}

Dyeing experiments were carried out on an AHIBA apparatus (AHIBA, Montreuil-Paris, France) with a liquor ratio of 1/100. Three types of dyes were used: a disperse dye (Blue Artisil BSQ), an acid dye (Red Nylosane E-BM) and a reactive dye (Yellow LANASOL 4G). We worked with a dye concentration of $1 \%$ owf (overweight fiber).

The fabrics were introduced into the dyebath at $30^{\circ} \mathrm{C}$. The temperature was then increased to $100{ }^{\circ} \mathrm{C}$ at a rate of $3{ }^{\circ} \mathrm{Cmin}^{-1}$. After $60 \mathrm{~min}$ at this temperature, the dyebath was cooled back to room temperature and recovered for colorimetric measurements. After calibration, the exhaustion of the dyeing bath was measured (spectrophotometer Jasco V530, Jasco, Bouguenais, France) by comparing the concentration of dyeing bath before and after dyeing.

Disperse dye is not very soluble in water. Our spectrophotometer measurements were made in a mixture of water and dimethyl formamide (50/50). We took this into account during the calibration step.

The wavelengths of disperse, acid and reactive dyes are 591, 435 and $395 \mathrm{~nm}$, respectively.

The structure of the three dyes used is given in Table 1.

\section{Wash-fastness tests}

The standard tests (ISO1 and ISO2) of wash fastness and staining were realized with a colorimeter Spectraflash SF 600 of Datacolor (Spectraflash, MontreuilParis, France), according to an evaluation scale from 0 to 5 . This evaluation is based on a comparison of the level of dyeing between samples before and after standard washings.

\section{RESULTS}

\section{PP finishing with CDs}

PP-knitted fabrics treated with a mixture of CTR and $\beta$-CD resulted in a weight increase of the samples. As shown in Figure 1, the degree of functionalization progressively increased with temperature in the range of $110^{\circ}-150^{\circ} \mathrm{C}$. The maximal rates were obtained at $140{ }^{\circ} \mathrm{C}$ 
(27\%wt). Figure 2 displays the time dependence of the reaction. The functionalization rate rapidly increased within the first 5 min of curing and reached a maximum value after $20 \mathrm{~min}$.

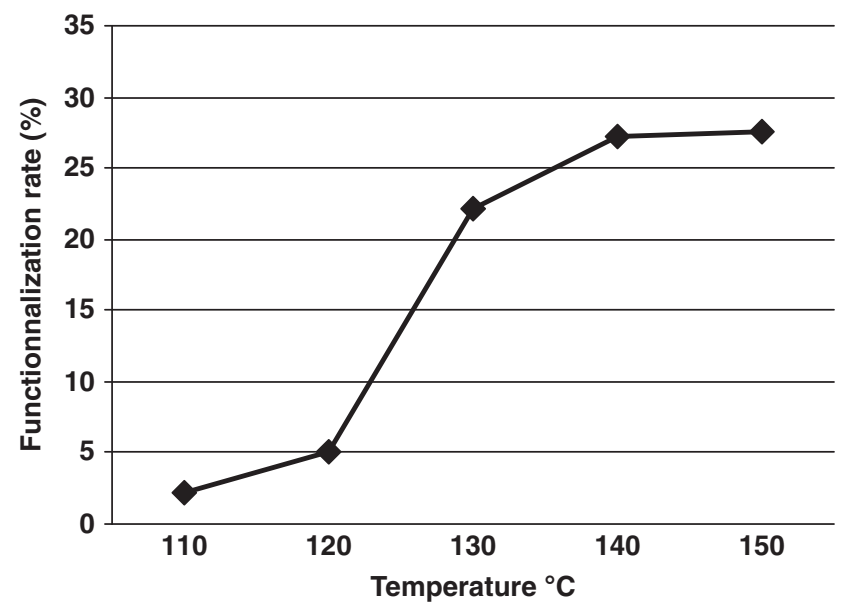

Figure 1 Influence of curing temperature on the functionalization rate of polypropylene-knitted fabric samples; curing time $=10 \mathrm{~min}$.

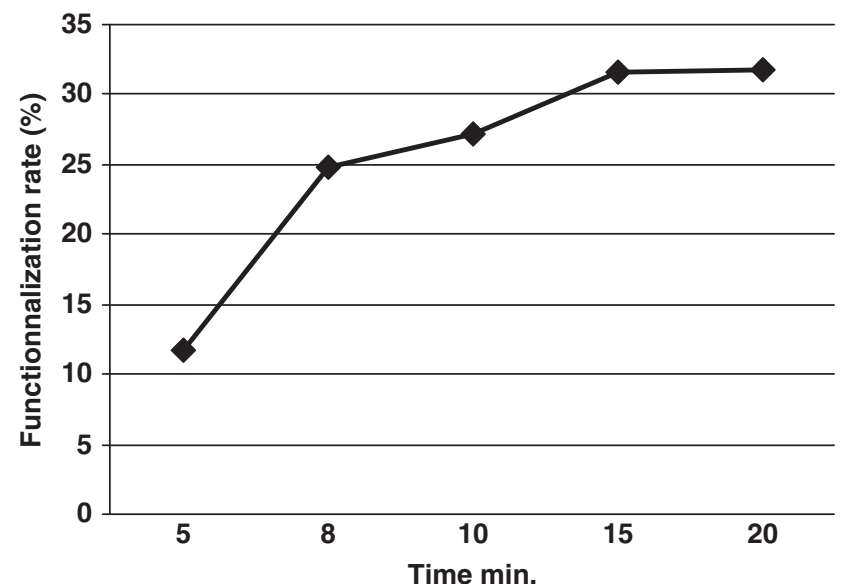

Figure 2 Influence of curing time on the functionalization rate of polypropylene-knitted fabric samples; curing at $140^{\circ} \mathrm{C}$.
A preliminary test showed that no reaction occurred when PP was treated with CTR alone. This confirmed that the functionalization of the support with $\beta-\mathrm{CD}$ occurred through the reaction between CTR and $\beta-\mathrm{CD}$ by polyesterification, giving rise to the formation of a crosslinked polymer of $\beta-\mathrm{CD}$ (named polyCTR$\beta$-CD hereafter) (Scheme 1), which formed a coating that was physically anchored (and not covalently grafted) to the fibers. Therefore, the modification of the fibers is expressed by the functionalization rate rather than by the grafting rate because the former is more appropriate.

\section{Damping tests}

PP surface modification on $\mathrm{CD}$ finishing was characterized by evaluating the contact angle of PP fibers with glycerol, using a Cahn balance instrument. The selected functionalized samples presented medium functionalization rates (14\%wt) and high functionalizing rates $(27 \% \mathrm{wt})$. These rates were obtained by fixing the temperature of treatment at $140{ }^{\circ} \mathrm{C}$ and the curing time at $10 \mathrm{~min}$ and by varying the concentration of CDs in the impregnating bath (Figure 3).

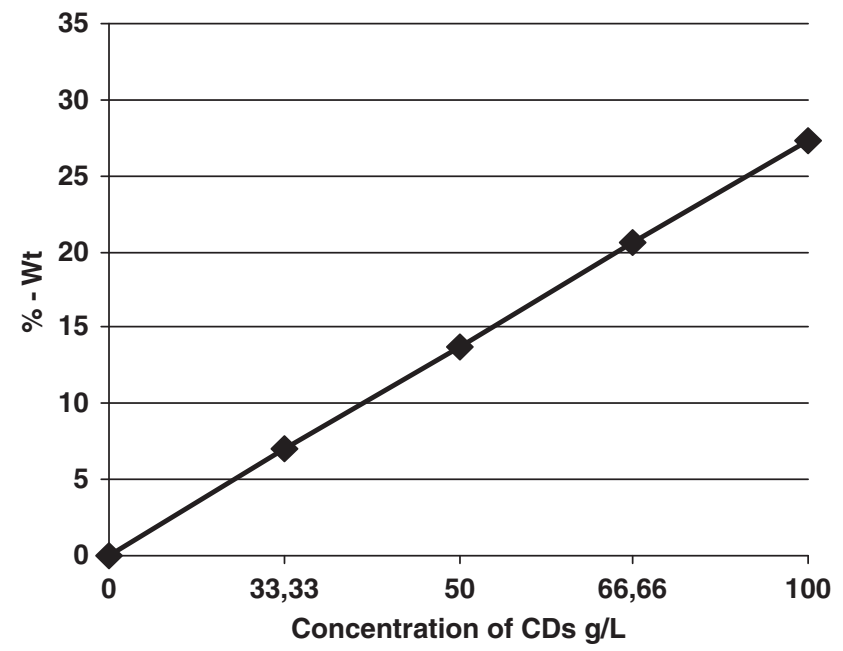

Figure 3 Functionalization rate of polypropylene-knitted fabric samples as a function of the concentration of cyclodextrins (CDs) in the impregnating bath; curing time $=10 \mathrm{~min}$ at $140^{\circ} \mathrm{C}$.

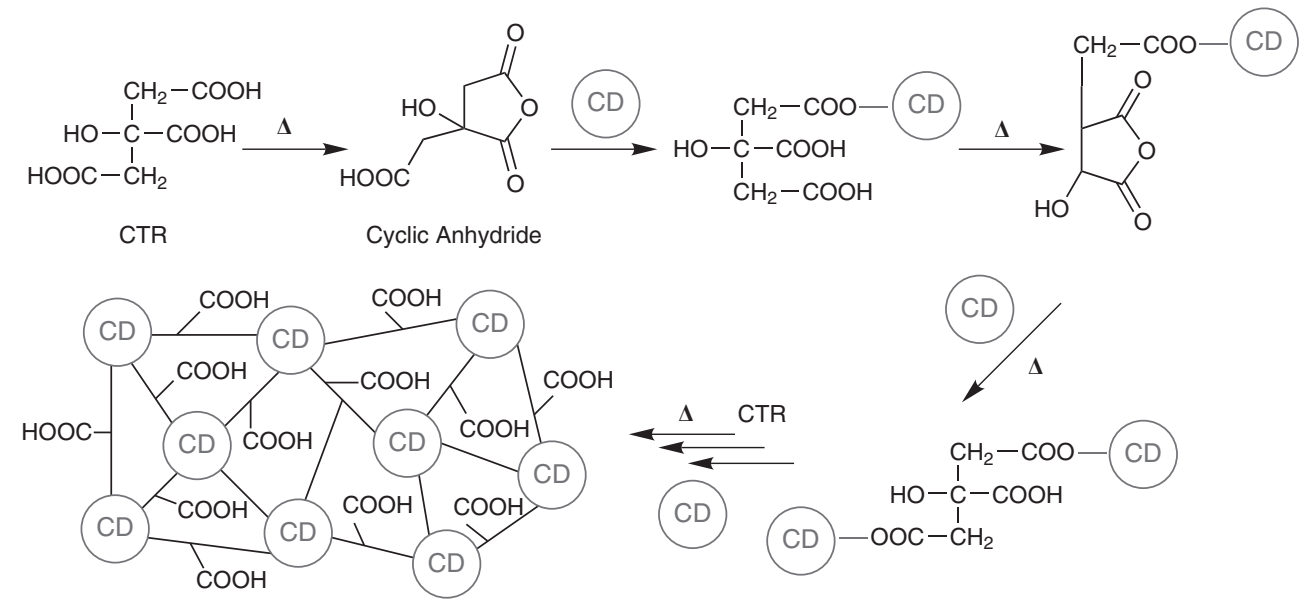

PolyCTR-CD

Scheme 1 Polymerization reaction between citric acid (CTR) and cyclodextrins (CDs): a three-dimensional polymer is obtained, consisting of CD cavities and $\mathrm{COOH}$ groups carried by crosslinking residues. 
a

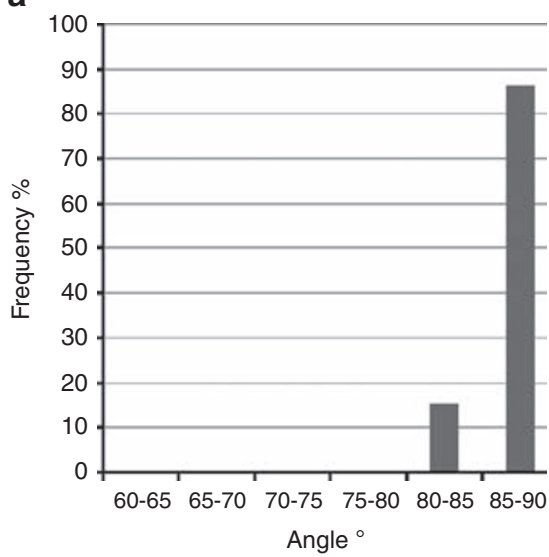

b

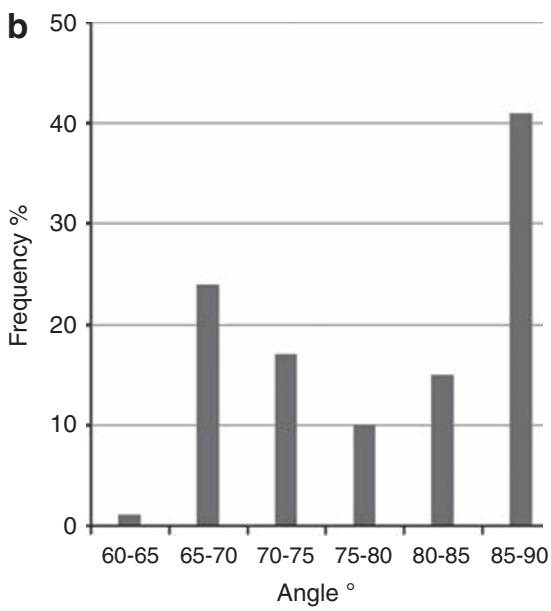

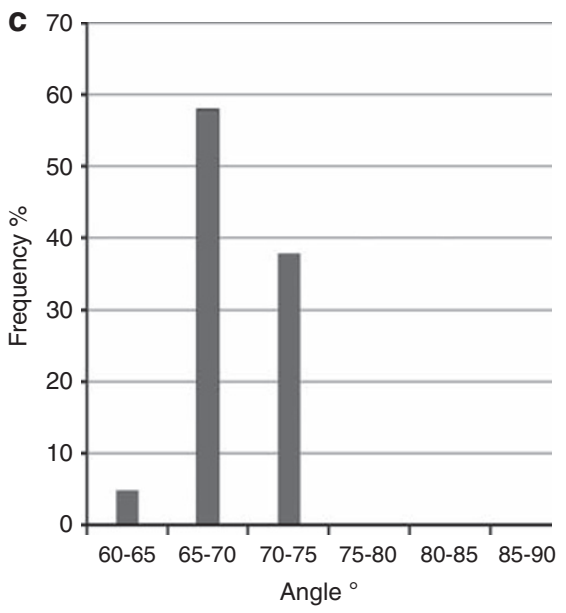

Figure 4 Distribution of wetting angles of polypropylene (PP) fibers, treated with $\beta$-cyclodextrin with different average functionalizing rates: 0,14 and $27 \%$ (respectively, a, b and $\mathbf{c}$ ).

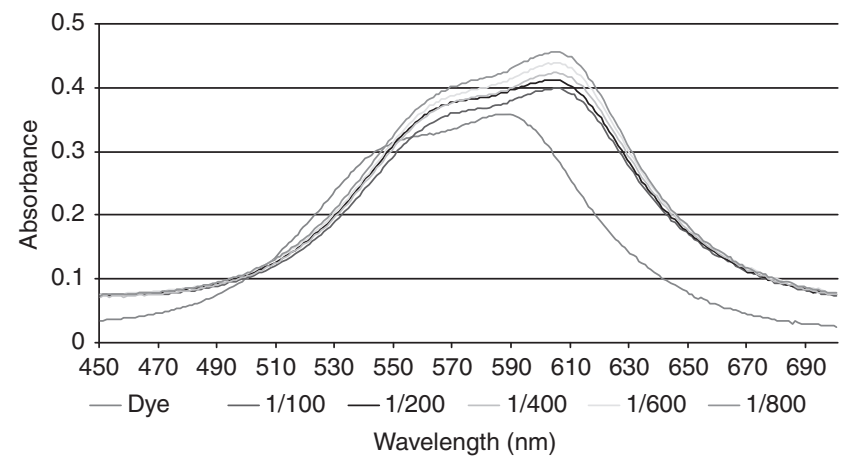

Figure 5 Visible spectrum of the disperse dye, Blue Artisil BSQ, in the presence of increasing $\beta$-cyclodextrin (CD) concentration. Molar ratios (dye)/ $(C D)=1 / 0-1 / 800$. A full color version of this figure is available at Polymer Journal online.

The histograms in Figure 4 show the statistical distribution of the contact angles measured on virgin and functionalized PP fibers. The virgin sample displayed a single distribution around $85-90^{\circ}$ (Figure 4a). PP with an intermediary functionalization rate presented a double distribution around $85-90^{\circ}$ and $65-70^{\circ}$ (Figure $4 \mathrm{~b}$ ). A single distribution around $65-70^{\circ}$ was observed for the highest functionalization rate (Figure 4c).

These results clearly display progressive surface coverage of the PP fibers on increasing modification rates by the hydrophilic polyCTR$\beta$-CD polymer.

\section{Complexation study between dyes and $\beta-C D$}

The spectrum of the disperse dye, Blue Artisil BSQ, was recorded in the presence of increasing molar ratios of $\beta$-CD by ultraviolet spectrophotometry in interval wavelengths ranging from 450 to $700 \mathrm{~nm}$. Dye concentration was fixed at $2 \cdot 10^{-5} \mathrm{moll}^{-1}$.

Figure 5 shows that the addition of $\beta-\mathrm{CD}$ provoked a modification of the spectrum of the disperse dye. The absorption band of the dye presented a maximum at $591 \mathrm{~nm}$, with a shoulder band at $544 \mathrm{~nm}$. In the presence of $\mathrm{CD}$, we noticed a bathochromic effect of $8 \mathrm{~nm}$, with a maximum at $609 \mathrm{~nm}$ and a shoulder band at $562 \mathrm{~nm}$. Besides, the increasing absorbance on $\mathrm{CD}$ addition confirmed that specific interactions such as a host-guest complex occurred between $\beta$-CD

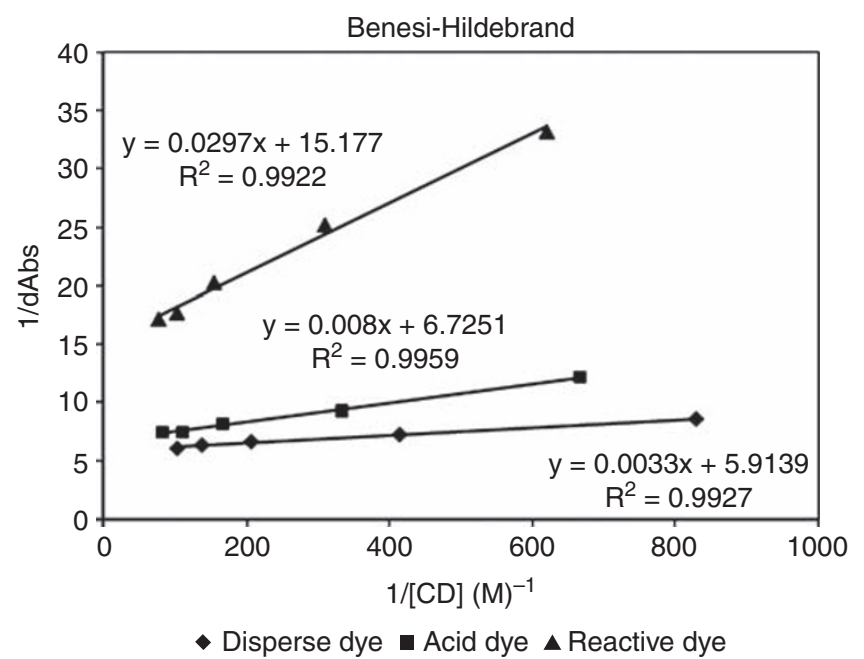

Figure 6 Benesi-Hildebrand plots for disperse, acid and reactive dyescyclodextrin systems.

and Blue Artisil BSQ. Despite the absence of a clear isosbestic point, the spectral changes of the substrates observed in Figure 5 (bathochromic+hyperchromic effects) in the presence of increasing amounts of CDs indicated inclusion of the substrate.

The Benesi-Hildebrand method was applied and resulted in the linear plots presented in Figure 6, which show $1 /(\Delta \mathrm{Abs})$ against $1 /$ (CD). The formation constants of the complexes were obtained from the ordinate at the origin and from the slope of each dye-CD system.

The calculated formation constants obtained from Figure 6 were 4303, 1526 and $2093 \mathrm{M}^{-1}$, respectively, for disperse dye, acid dye and reactive dye. These data confirm that all three dyes can be included into the free $\beta-C D$ cavities in aqueous solutions. It is worth mentioning that these constants should not be extrapolated to $\beta-C D$ that is immobilized onto fibers because, in solid-liquid heterogeneous systems, the $\beta-C D$ cavities that are present in the polyCTR- $\beta$-CD coating present a different accessibility. In conclusion, this series of experiments only shows the possibility of the host-guest inclusion between $\beta-C D$ and dyes. In fact, despite our attempts involving solid nuclear magnetic resonance experiments, we could not directly show the inclusion of any substrate into $\beta$-CD fixed on solid supports because 

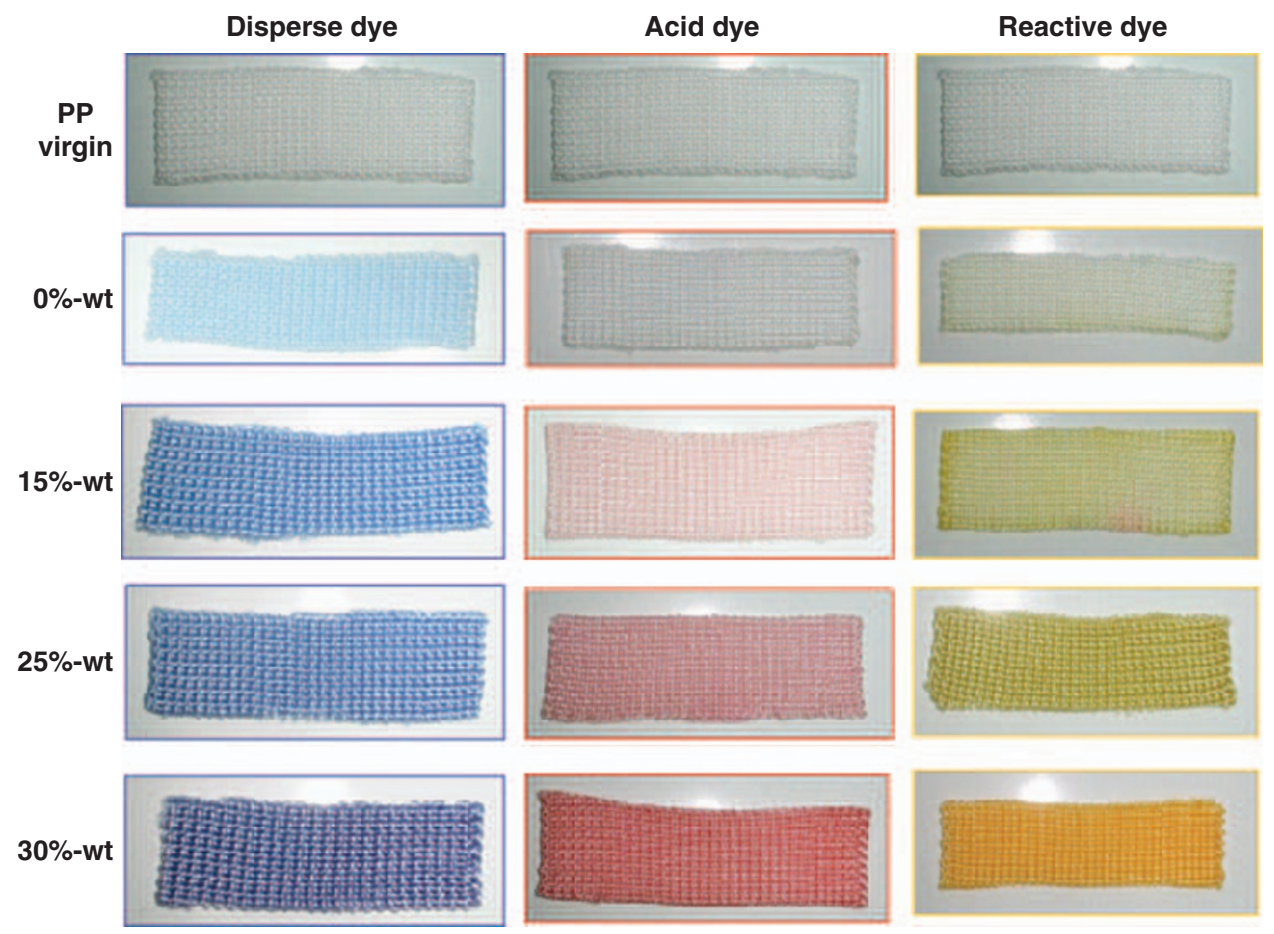

Figure 7 Polypropylene (PP) samples with different rates of functionalization in cyclodextrin, dyed with disperse, acid and reactive dyes and washed according to the standard test, ISO1.

of the insufficient sensitivity and resolution of this technique. Surface plasmon resonance is often used in literature to show the adsorption of substrates onto a CD functionalized surface. However, this technique involves a model surface that does not really correspond to a textile surface. In conclusion, there exists no direct technique that can be adapted to detect efficient inclusion complexation onto textiles. Therefore, only indirect approaches are possible. ${ }^{17}$

\section{Dyeing tests}

PP fabrics that were finished with variable functionalization rates (from 15 to $30 \% \mathrm{wt}$ ) were prepared by varying the concentration of $\beta$ $\mathrm{CD}$ in the impregnating bath with a temperature and curing time of $140{ }^{\circ} \mathrm{C}$ and $15 \mathrm{~min}$, respectively. All samples were treated with disperse, acid and reactive dye according to the method described above.

Figure 7 shows the evolution of the color intensity. The results obtained show a significant difference in the dyeing level between the virgin $\mathrm{PP}$ and the $\mathrm{PP}$ treated with $\beta-\mathrm{CD}$.

Direct visualization of the different samples in Figure 7 shows enhancement of the dye shade of PP after its treatment with $\beta$-CD. We noticed that the dye level increased with the quantity of $\beta-\mathrm{CD}$ fixed onto the PP samples.

In the cases of higher functionalization rates, it was important to notice the fine homogeneous levelness-the term most appropriate seems to be 'unison'- to specify that the color is homogeneous on the whole surface of the sample when we dye using the three classes of dyes. This confirms the good and homogeneous repartition of polyCTR- $\beta-\mathrm{CD}$ onto the PP-knitted samples.

These results are confirmed by Figure 8, in which the exhaustion rate of the dyeing baths is correlated with the degree of functionalization of the knitted fabric with $\beta$-CD. Figure 8 also shows that the exhaustion rate of the dyeing bath increased with the quantity of CDs fixed onto the PP samples for the three types of dye used. For disperse and reactive dyes, results reveal that below a threshold value of the

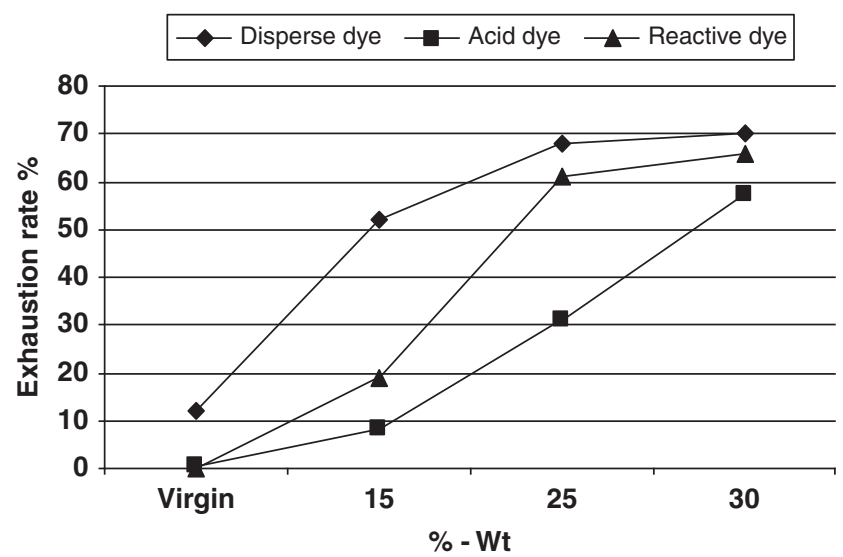

Figure 8 Exhaustion rates of the dyeing baths with functionalization rates (\%wt) of PP-knitted fabrics finished with cyclodextrin (CD) $\beta$-CD dyes with Blue Artisil $\bullet$; Red Nylosane $\mathbf{\square}$; Yellow Lanasol $\boldsymbol{\Delta}$

quantity of $\mathrm{CD}$ onto PP fibers, the exhausted quantity in the dyeing bath becomes constant. This signifies the presence of equilibrium between the solubility of the dyes in the bath and their substantivity toward the fiber (with an initial dye concentration of $1 \%$ ). The exhaustion rate can be improved by increasing the dye concentration in the dyeing bath. In the case of acid dye, we observed an almost linear relation between the exhaustion rate and the functionalization rate. This full bath dyeing was very practical without any addition of auxiliary products, reveals good rates of dyeing and shows great affinity with a good dyeing levelness.

\section{Wash fastness}

The results of the standard tests (ISO1 and ISO2) used to evaluate the resistance of the dyed samples toward washings at 40 and $60{ }^{\circ} \mathrm{C}$ are 
reported in Table 2. With regard to shade change, the measured level remained optimal (4-5) after one washing at $40{ }^{\circ} \mathrm{C}$ for $30 \mathrm{~min}$ and decreased slightly to 4 after washing at $60{ }^{\circ} \mathrm{C}$. This indicates good resistance of all three dyes to washings on the textiles treated with CD.

With regard to the staining standard tests, Table 2 also shows that the virgin samples washed in the presence of $\mathrm{CD}$-finished dyed samples did not undergo staining during the washing tests. These results, with the reactive dye (level 5) for the two standard tests, may indicate a strong binding of this dye with fibers through covalent bonds.

\section{DISCUSSION}

In this paper, we show that PP modification with CD under controlled conditions allows for control of the dyeability of this type of textile. As a matter of fact, because of the versatile properties of the fixed polyCTR-CD polymer on the fabric, it was possible to control the dyeing intensity of the support.

Table 2 Different results of washing fastness tests on the $\beta$-cyclodextrin functionalized PP samples after dyeing with the three classes of dyes

\begin{tabular}{lccccc}
\hline & \multicolumn{2}{c}{$40^{\circ} \mathrm{C}-30 \mathrm{~min}$} & & \multicolumn{2}{c}{$60^{\circ} \mathrm{C}-30 \mathrm{~min}$} \\
\cline { 2 - 3 } \cline { 5 - 6 } Dyes & Shade change & Staining & & Shade change & Staining \\
\hline Dispersed dye & $4-5$ & 5 & & 4 & $4-5$ \\
Acid dye & $4-5$ & 5 & & $4-5$ & $4-5$ \\
Reactive dye & $4-5$ & 5 & & 4 & 5 \\
\hline
\end{tabular}

First, the possibility of forming an inclusion complex between $\beta$-CD and the disperse dye, an increase in the exhaustion rate of the dyeing bath with functionalized samples, direct visualization of a dyeing rate that was improved with a good dyeing evenness, as well as the good results of washing fastness, could confirm the efficacy of the finishing reaction with the aim of dyeing PP by the classical bath-dyeing method. Figure 9 illustrates the various interactions between PP fibers functionalized by $\beta-\mathrm{CD}$ and the disperse dye.

The possibility of encapsulating the acid dye into the $\beta$-CD cavity, the presence of various interactions (ionic and hydrogen bonding) between the hydroxyl groups of $\beta-\mathrm{CD}$, the carboxylate functions on PolyCTR- $\beta-C D$, as well as the auxochrom groups of acid dye, can explain the increase in the exhaustion rate and the good results of washing fastness. Figure 10 summarizes these various interactions between acid dye and the functionalized PP samples.

The possibility of dyeing PP with reactive dye with good washing results can be explained by the presence of a complexation between the dye molecule and $\beta-C D$, and also by the possibility of the formation of covalent bonding by reaction between hydroxyl groups of $\beta-C D$ and the reactive halogenated groups of the dye, in addition to the ionic bonding between the carboxylate groups of polyCTR- $\beta-\mathrm{CD}$ and protonated amino and imino groups of the reactive dye. The various possible interactions between reactive dye and PP functionalized with $\beta$-CD are illustrated in Figure 11.

As observed in the spectrophotometric and wash fastness studies, the sulfonate groups present on the structures of Red Linosan and Yellow Lanasol do not prevent their inclusion complexation into the CD cavity. As a matter of fact, previous works showed that benzene rings substituted with sulfonate groups may undergo inclusion inside

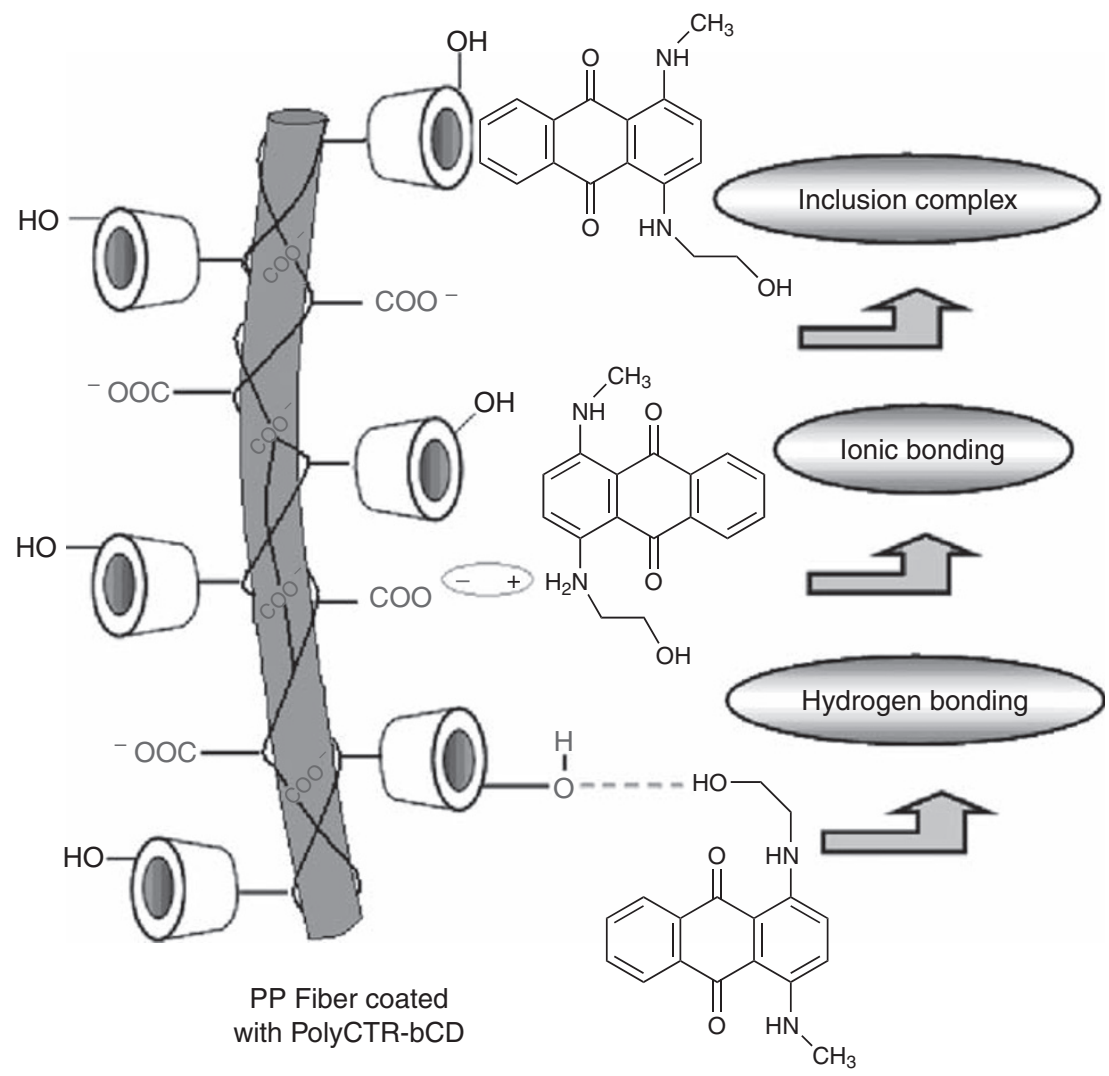

Figure 9 Illustration of the different interactions between disperse dye, Blue Artisil BSQ and polypropylene (PP) functionalized with polyCTR- $\beta$-CD. CTR, citric acid. A full color version of this figure is available at Polymer Journal online. 


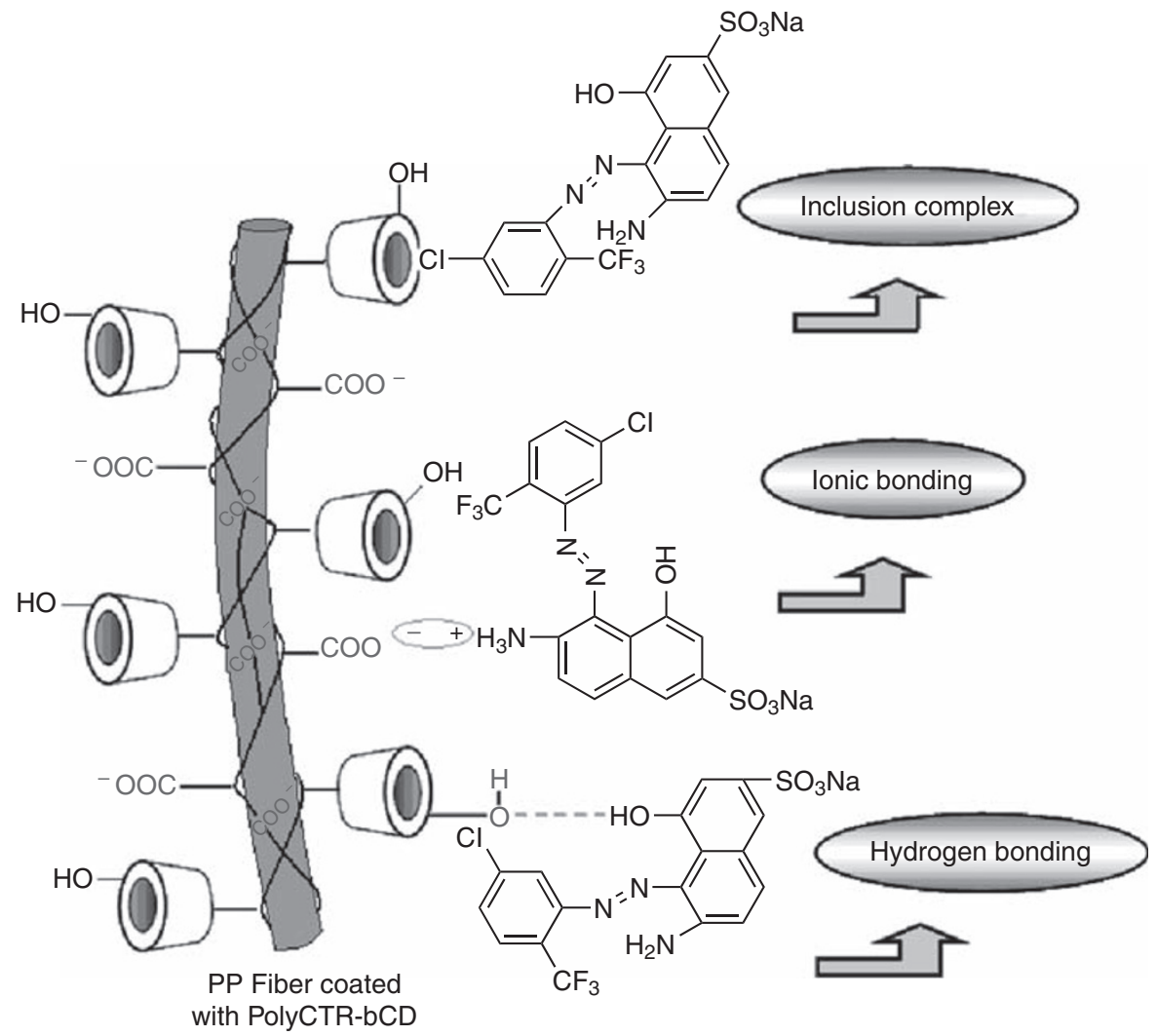

Figure 10 Illustration of the different interactions between acid dye, Red Nylosane E-BM and polypropylene (PP) functionalized with polyCTR- $\beta$-CD. CTR, citric acid. A full color version of this figure is available at Polymer Journal online.

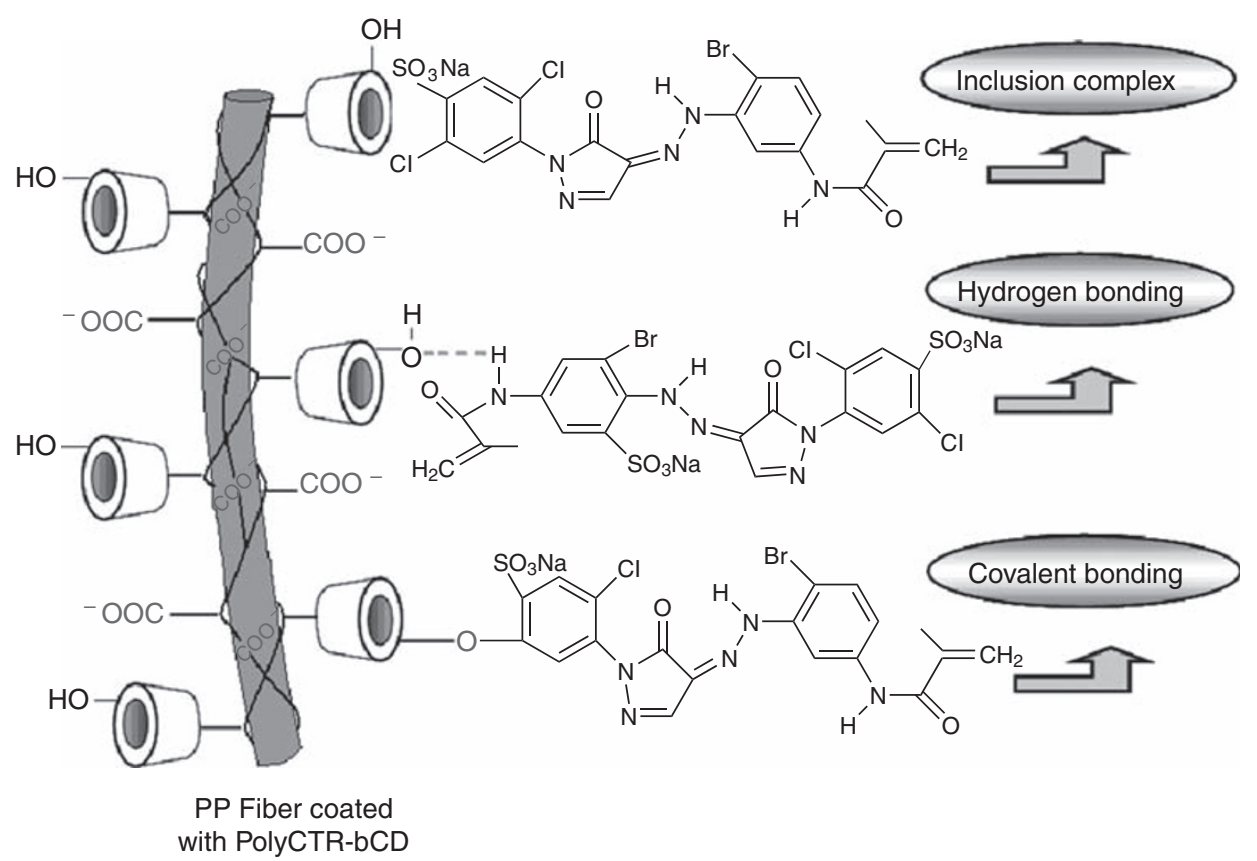

Figure 11 Illustration of the different interactions between reactive dye, Yellow LANASOL 4G and polypropylene (PP) functionalized with polyCTR- $\beta$-CD. A full color version of this figure is available at Polymer Journal online. 
CDs. ${ }^{18-20}$ In these cases, the sulfonate groups protrude from the $\mathrm{CD}$ macrocycle, whereas the apolar benzene ring deeply penetrates the cavity.

Besides, our experimental results also displayed that the sulfonated groups carried by these dyes (Red Linosan and Yellow Lanasol) did not provoke repulsive interactions with the residual carboxylate groups present on the polyCTR- $\beta$-CD fixed onto the PP support. As a matter of fact, in the case of Red Nylosane, it can be observed in Figure 10 that the sulfonate group is situated in the position opposite from the groups that exert attractive interactions with the support: amino group and aromatic ring. Therefore, if such a repulsion effect occurred, this sulfonate group would not prevent sorption of the dye onto the support. With regard to Yellow Lanasol, indeed the sulfonate group carried by the dye seems to be situated in a position that would involve a steric proximity with the carboxylate groups carried by the CD polymer (especially in the case of the covalent binding mechanism and inclusion complex mechanism described in Figure 11).

\section{CONCLUSION}

In this paper, we reported that PP fabrics could be finished with controlled rates of CDs through a crosslinking reaction that could be carried out under a pad-dry-cure process, in environmentally friendly conditions.

The surface modification of PP fibers with polyCTR-CD markedly enhanced their dyeability with three dyes belonging to different classes, using a classical bath-dyeing method, avoiding more costly and complicated processes such as plasma pretreatment. We could observe that the dyeing intensity was correlated with the degree of functionalization of the PP fabric.

The observed enhancement of dye uptake was attributed to the encapsulation of dyes in the $\beta$-CD cavities on the one hand and to other types of interactions, such as acid-base, hydrogen bonding or even covalent bonding, with the polyCTR- $\beta \mathrm{CD}$ network in the case of a reactive dye on the other hand. This explains why the efficiency of the dyeing experiments did not strictly depend on the class of dye.

It was observed that the dyeing level depended on the modification rate of PP fibers with $\mathrm{CD}$. The good fastness observed also showed the effectiveness of this concept of textile surface modification.
1 Shao, Y, Martel, B \& Morcellet, M Weltrowski MInteraction between beta-cyclodextrin water-soluble dyes. Canadian Textile J 53-58 (1996).

2 Szejtli, J. Cyclodextrins in the textile industry. Starch/Stärke 55, 191-196 (2003).

3 Shah, C. D. \& Jain, D. K. Dyeing of modified polypropylene: cationic dyes on chlorinated polypropylene. Textile Res J 53, 274-281 (1983).

4 Sengupta, A. K., Sen, K. \& Mukhopadhyay, A. False twist texturization of polypropylene multifilament yarns part IV: Structural influences on dye uptake. Textile Res J 56, 511-515 (1986)

5 Rowland, S. P., Welch, C. M, Brannan, M. A. F. \& Dudley, M. Gallagher introduction of ester cross links into cotton cellulose by a rapid curing process. Textile Res J 37, 933-941 (1967).

6 Buschmann, H. J., Knittel, D. \& Schollmeyer, E. Cyclodextrins as levelling agents for high-temperature dyeing of polyester. TPI. Text. Prax. Int. 45, 376-378 (1990).

7 Savarino, P., Viscardi, G., Quagliotto, P., Montoneri, E. \& Barni, E. Reactivity and effects of cyclodextrins in textile dyeing. Dyes pigm. 42, 143-147 (1999).

8 Reuscher, H., Hirsenkom, H. \& Haas, W. EP 0697415A1 (1996).

9 Moldenhauer, J. P. \& Reuscher, H. Textile finishing with MCT- $\beta$-cyclodextrin, In: (eds. Torres Labandeira, J.J., Vila-Jato, J. L.). Proc 9th Symp Cyclodextrins (1999) 161-165, (Kluwer Academic Publishers, Dordrecht, 1998).

10 Le Thuaut, Ph., Martel, B., Crini, G., Maschke, U., Coqueret, X. \& Morcellet, M. Grafting of cyclodextrins onto polypropylene nonwoven fabrics for the manufacture of reactive filters- Part 1: Synthesis parameters. J. Appl. Polym Sci. 77, 2118-2125 (2000).

11 Martel, B., Weltrowski, M., Ruffin, D. \& Morcellet, M. Polycarboxylic acids as crosslinking agents for grafting cyclodextrins onto cotton or wool fabrics. J. Appl. Polym. Sci. 83, 1449-1456 (2002).

12 Martel, B., Morcellet, M., Ruffin, D., Ducoroy, L. \& Weltrowski, M. Finishing of polyester fabrics by cyclodextrins by using polycarboxylic acids as crosslinking agents. J. Incl. Phenom. Macrocycl. Chem. 44, 443-446 (2002).

13 El Ghoul, Y., Martel, B., Morcellet, M., Campagne, C., El Achari, A. \& Roudesli, S. Mechanical and physico-chemical characterization of cyclodextrin finished polyamide fibers. J. Incl. Phenom. Macrocycl. Chem. 57, 47-52 (2007).

14 Laurent, T., Blanchemain, N., Haulon, S., Neut, C., Hildebrand, H F., Morcellet, M. \& Martel, B. Cyclodextrin finishing of polypropylene mesh as artificial abdominal wall support implant for the improved delivery of ciprofloxacin. Proc of the second edition of the "International Conference of Applied Research on Textile" CIRAT, November 2006, Monastir, Tunisia, 2006.

15 Berg, J. C. 'Wettability' Surfactant Science Series, (Marcel Dekker, New York, 1993).

16 Shao, Y., Weltrowski, M., Morcellet, M. \& Martel, B. Interaction between betacyclodextrin and water-soluble dyes. Canadian Textile J. 113, 53-56 (1996).

17 Leclercq, L., Bria, M., Morcellet, M. \& Martel, B. B-cyclodextrin-polyelectrolyte interactions in aqueous solutions: I Example of poly(4-sodium styrenesulfonate) (NaPSS), J. Incl. Phenom. Molec. Recogn. Chem. 30, 215-225 (1998).

18 Bergeron, R. J. \& Roberts, W. P. Boundary conditions for the Benesi-Hildebrand. Analytical Biochem. 90, 844-848 (1978).

19 Zhang, H., Chen, G., Wang, L., Ding, L., Tian, Y., Jin, W. \& Zhang, H. Study on the inclusion complexes of cyclodextrin and sulphonated azo dyes by electrospray ionization mass spectrometry. Int. J. Mass Spectr. 252, 1-10 (2006).

20 Caron, L., Tilloy, S., Monflier, E., Wieruszeski, J. M., Lippens, G., Landy, D., Fourmentin, S. \& Surpateanu, G. Study of the inclusion complexes of $\beta$-cyclodextrin with the sodium salt of trisulfonated triphenylphosphine. J. Incl. Phenom. Macr. Chem $\mathbf{3 8}$, 361-379 (2000). 